\title{
Effects of Radiation Therapy on Established Neurogenic Heterotopic Ossification
}

\author{
Chan Ho Lee, MD', Su Jung Shim, MD², Hyun Jung Kim, MD¹ , Hyuna Yang, MD¹, Youn Joo Kang, MD
}

Departments of ${ }^{1}$ Rehabilitation Medicine, ${ }^{2}$ Radiation Oncology, Eulji Hospital, Eulji University School of Medicine, Seoul, Korea

Heterotopic ossification (HO) is frequently seen on rehabilitation units after spinal cord injuries, fractures, brain injuries, and limb amputations. Currently, there is no effective treatment for HO other than prophylaxis with anti-inflammatory medications, irradiation, and bisphosphonate administration. These prophylactic treatments are not effective for managing ectopic bone once it has formed. Here we describe three cases of established neurogenic HO treated with radiation therapy (RT). All patients had decreased serum alkaline phosphatase (ALP) and bone-specific ALP levels with decreased pain but increased range of motion immediately after RT. Post-treatment X-rays revealed no further growth of the HO. All patients maintained clinical and laboratory improvements 4 or 6 months after the RT. Our results suggest that RT is safe and effective in decreasing pain and activity of neurogenic HO.

Keywords Brain injuries, Heterotopic ossification, Radiotherapy

\section{INTRODUCTION}

Heterotopic ossification (HO) is mature lamellar bone formed in soft tissue. HO has been documented in patients with spinal cord injuries, burns, fractures, brain injury, and limb amputations [1].

Several laboratory tests have been used to determine whether their ability in predicting or identifying the formation of HO, including serum alkaline phosphatase (ALP) levels [2]. Bone-specific alkaline phosphatase
(BALP) level has been proven to be a sensitive and reliable indicator of bone metabolism [3]. When $\mathrm{HO}$ is accompanied by joint pain and ankylosis, it can limit rehabilitation. Therefore, it is important to treat HO. Radiation therapy (RT) has been found to have prophylactic effect against HO [4]. However, only a few reports are available on its short-term therapeutic effects in established HO [5,6]. Here, we report the results of 4-6 month follow-up evaluation for three patients with $\mathrm{HO}$ after brain injury who have undergone RT. For the first time,

Received April 25, 2016; Accepted June 7, 2016

Corresponding author: Youn Joo Kang

Department of Rehabilitation Medicine, Eulji Hospital, Eulji University School of Medicine, 68 Hangeulbiseok-ro, Nowon-gu, Seoul 01830, Korea. Tel: +82-2-970-8315, Fax: +82-2-979-8268, E-mail: md52516@hanmail.net

ORCID: Chan Ho Lee (http://orcid.org/0000-0002-5129-6652); Su Jung Shim (http//orcid.org/0000-0002-2364-6651); Hyun Jung Kim (http//orcid. org/0000-0002-2198-5668); Hyuna Yang (http//orcid.org/0000-0002-2265-4166); Youn Joo Kang (http://orcid.org/0000-0002-9938-5435).

(c) This is an open-access article distributed under the terms of the Creative Commons Attribution Non-Commercial License (http://creativecommons.org/ licenses/by-nc/4.0) which permits unrestricted noncommercial use, distribution, and reproduction in any medium, provided the original work is properly cited. Copyright ( 2016 by Korean Academy of Rehabilitation Medicine 
we serially evaluated the changes in the range of motion (ROM) of the involved joints, pain severity, and serum bone formation markers (BALP) after RT.

\section{CASE REPORTS}

\section{Case 1}

A 23-year-old man with a diffuse axonal brain injury and multiple fractures caused by a fall 1 year earlier was admitted to Department of Rehabilitation Medicine, Eulji Hospital. Brain magnetic resonance imaging (MRI) results are shown in Fig. 1. He had undergone open reduction and internal fixation for left distal tibia and fibula, right calcaneus, and right proximal tibia fractures in the Department of Orthopedic Surgery. After the injury, he suffered from bilateral lower extremity weakness with moderate cognitive impairment (Korean-Mini Mental State Examination score $=22$ ). HO was diagnosed at the right hip and both knee joints at 6 months after the accident (Fig. 2). At this point, his serum ALP level and BALP level were $119 \mathrm{IU} / \mathrm{L}$ (normal range, 80-270 IU/L) and $22.3 \mathrm{IU} / \mathrm{L}$ (normal range, 4.5-16.9 IU/L), respectively. Although he had been taking $600 \mathrm{mg}$ oral etidronate disodium daily for 6 months and undergoing physical therapy, the passive ROM of the involved joints was limited. He complained of pain aggravated by ROM exercise (visual analog scale [VAS] score $=8$ ). One year after the accident, his serum ALP level and BALP level were progressively elevated to $213 \mathrm{IU} / \mathrm{L}$ and $67.6 \mathrm{IU} / \mathrm{L}$, respectively. To relieve the pain, the patient underwent 10 days of RT for a total radiation dose of $20 \mathrm{~Gy}$ in $2 \mathrm{~Gy}$ fractions on the right hip and both knee, respectively. After completing the RT, he had mild improvement in joint ROM with decreased pain on exercise (Table 1). He had no side effect such as bone marrow suppression. His serum ALP and BALP levels were decreased immediately after the RT to $172 \mathrm{IU} / \mathrm{L}$ and $48.6 \mathrm{IU} / \mathrm{L}$, respectively (Fig. 3). Six months after the RT, he maintained both clinical and laboratory improvements (Table 1, Fig. 3).

\section{Case 2}

A 52-year-old man who had been diagnosed with a meningioma 12 years earlier was admitted to Department of Rehabilitation Medicine, Eulji Hospital. Brain MRI results are shown in Fig. 1. He had undergone craniotomies and tumor removal at the Department of Neurosurgery twice (12 and 9 years earlier). He had progressive weakness of all four extremities with loss of ambulation. He was diagnosed with $\mathrm{HO}$ in both hip joints (Fig. 2) approximately at 3 months before admission to our rehabilitation department. Although the patient had been taking $1,200 \mathrm{mg}$ of oral etidronate disodium daily and undergone physical therapy, his passive ROM was limited and he complained of pain (VAS=6). His serum ALP and BALP levels were elevated to $289 \mathrm{IU} / \mathrm{L}$ and $92.1 \mathrm{IU} / \mathrm{L}$, respectively, at the time of admission. The patient was given 10 days of RT to relieve pain in a total radiation dose of $20 \mathrm{~Gy}$ with $2 \mathrm{~Gy}$ per fraction on both hips, respectively. After RT, his ROM was improved and his pain was decreased (Table 1). His serum ALP and BALP levels were decreased immediately
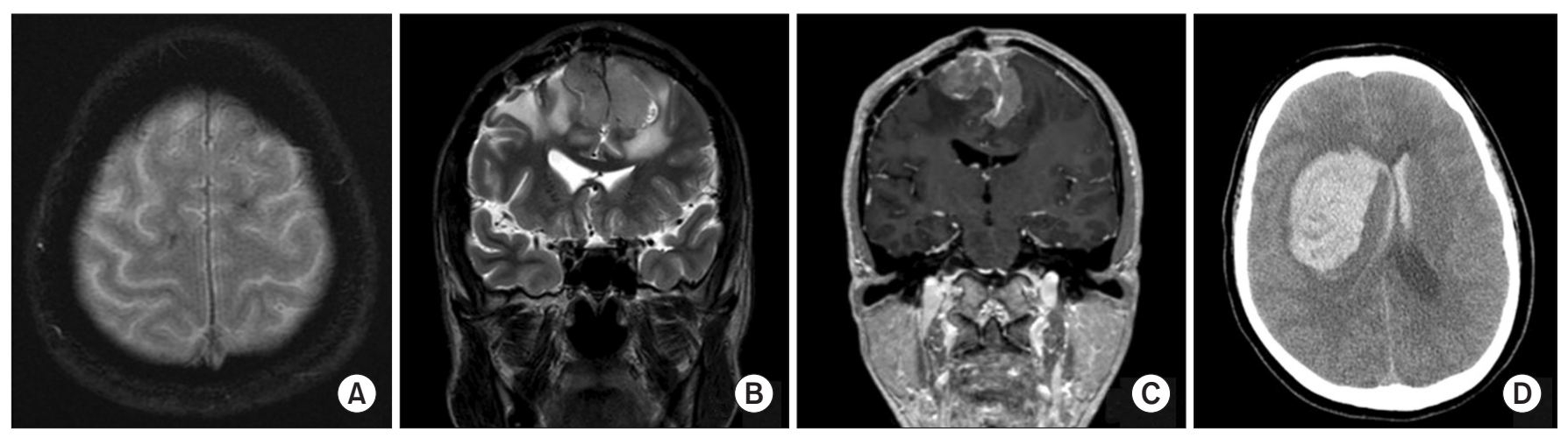

Fig. 1. (A) Gradient recalled echo brain magnetic resonance imaging (MRI) in case 1 showing a dark signal intensity at the gray/white matter interfaces in the bilateral superior frontal gyri suggestive of diffuse axonal injury. In case 2, brain MRI showing an enhancing mass at the falx (B) and in the parietal area with a cerebrospinal fluid cleft suggestive of extra-axial meningioma (C). (D) In case 3, brain computed tomography showing an intracerebral hemorrhage in the right basal ganglia and an intraventricular hemorrhage. 

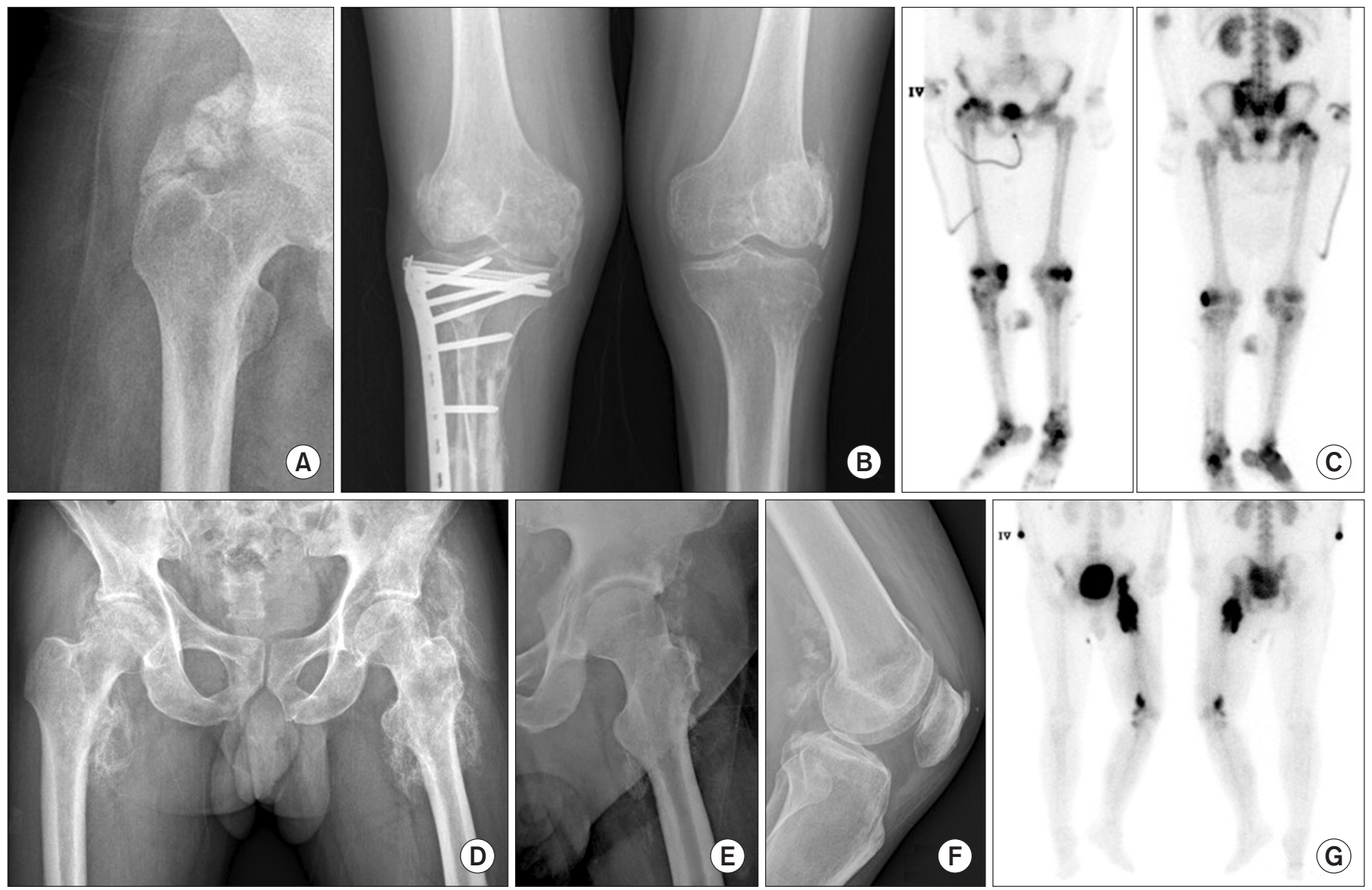

Fig. 2. In case 1, AP radiographs of the right hip (A) and both knees showing HO (B). Whole body bone scan showing increased uptake at the right hip and both knees (C). (D) In case 2, AP radiographs of both hips showing HO. In case 3, AP radiograph of the left hip (E) and lateral radiograph of the left knee showing HO (F). Whole body bone scan showing increased uptake at the left hip and knee (G). AP, anteroposterior; HO, heterotopic ossification.

Table 1. Changes in VAS scores and passive range of motion in the three patients after RT

\begin{tabular}{|c|c|c|c|c|c|c|}
\hline & & Pre RT & Post RT & $\begin{array}{c}\text { Post RT } \\
(1 \mathrm{mo})\end{array}$ & $\begin{array}{c}\text { Post RT } \\
(4 \mathrm{mo})\end{array}$ & $\begin{array}{c}\text { Post RT } \\
(6 \mathrm{mo})\end{array}$ \\
\hline \multirow[t]{4}{*}{ Case 1} & Rt hip flexion $\left({ }^{\circ}\right)$ & 90 & 100 & 100 & 100 & 100 \\
\hline & Rt knee flexion $\left({ }^{\circ}\right)$ & 90 & 100 & 100 & 100 & 100 \\
\hline & Lt knee flexion $\left({ }^{\circ}\right)$ & 80 & 90 & 90 & 90 & 90 \\
\hline & VAS & 8 & 4 & 4 & 4 & 4 \\
\hline \multirow[t]{3}{*}{ Case 2} & Rt hip flexion $\left({ }^{\circ}\right)$ & 65 & 90 & 90 & 90 & NA \\
\hline & Lt hip flexion $\left({ }^{\circ}\right)$ & 50 & 70 & 70 & 70 & NA \\
\hline & VAS & 6 & 4 & 4 & 4 & NA \\
\hline \multirow[t]{3}{*}{ Case 3} & Lt hip flexion $\left(^{\circ}\right)$ & 90 & 90 & 90 & 90 & 90 \\
\hline & Lt knee flexion $\left(^{\circ}\right)$ & 100 & 110 & 110 & 110 & 110 \\
\hline & VAS & 6 & 4 & 3 & 3 & 3 \\
\hline
\end{tabular}

VAS, visual analog scale; RT, radiation therapy; NA, not applicable. 

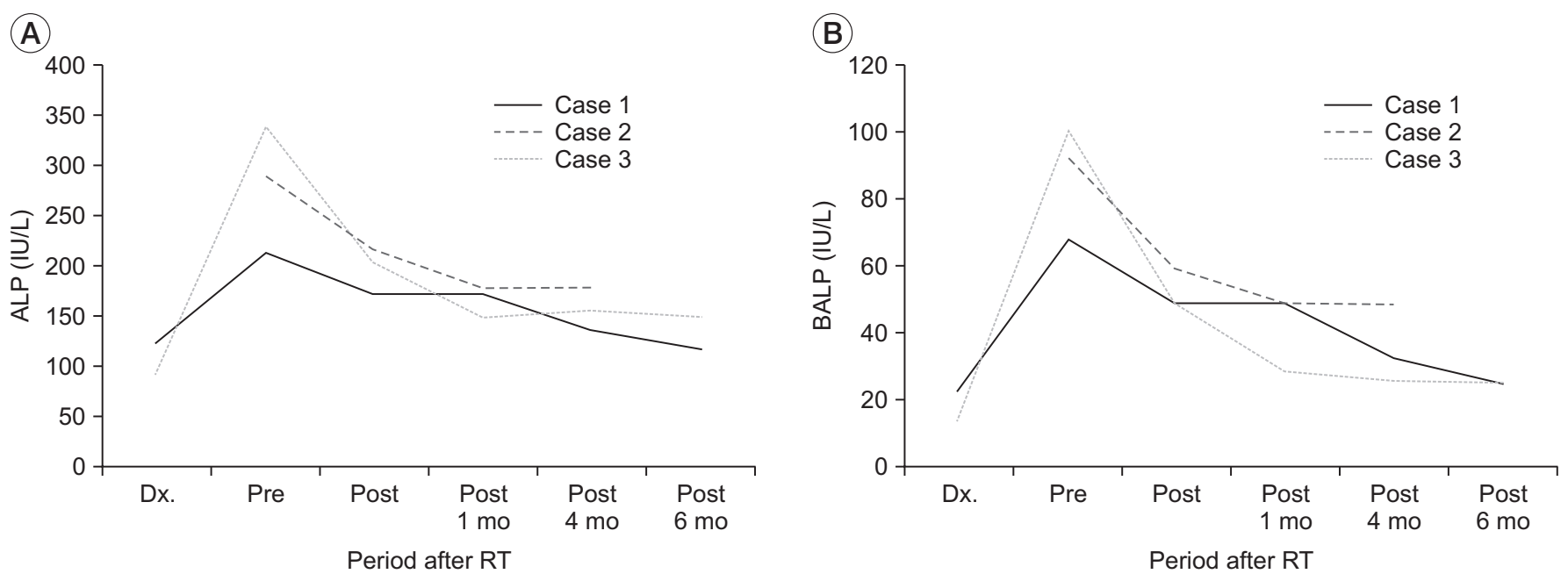

Fig. 3. (A) Serum alkaline phosphatase (ALP) and (B) bone-specific alkaline phosphatase (BALP) levels are dramatically decreased immediately after radiation therapy (RT) in all three cases. These values remained low or decreased further throughout the follow-up period. Dx, the time of the initial diagnosis of heterotopic ossification.

after RT to $216 \mathrm{IU} / \mathrm{L}$ and $58.7 \mathrm{IU} / \mathrm{L}$, respectively (Fig. 3). After RT, he developed leukocytopenia $(3,130 / \mu \mathrm{L})$, which had normalized by 1 month after RT. After 4 months, his improvement was maintained (Table 1, Fig. 3). At that time, he was diagnosed with recurrent meningioma. Therefore, he underwent reoperation.

\section{Case 3}

A 35-year-old man with a spontaneous right intracranial/intraventricular hemorrhage (Fig. 1) 6 months earlier was admitted to Department of Rehabilitation Medicine, Eulji Hospital. At the time of injury, he had undergone burr hole trephination and closed drainage in the Department of Neurosurgery. He suffered from left hemiplegia. HO was developed at the left hip and knee joints (Fig. 2 ) at 3 months after the stroke. Although he had been taking oral etidronate disodium daily and receiving physical therapy, he developed contractures and complained of pain on exercise (VAS=6). Five months after the stroke, his serum ALP and BALP levels were progressively elevated to $340 \mathrm{IU} / \mathrm{L}$ and $101 \mathrm{IU} / \mathrm{L}$, respectively. At that point, to relieve pain, the patient was given 10 days of RT for a total radiation dose of $20 \mathrm{~Gy}$ in $2 \mathrm{~Gy}$ fractions left hip and knee, respectively. Immediately after completing the RT, he only had mild improvement of the ROM in the left knee joint with decreased pain (Table 1). He had no side effect such as bone marrow suppression. Immediately after RT, his serum ALP and BALP levels were decreased to $203 \mathrm{IU} / \mathrm{L}$ and $47.9 \mathrm{IU} / \mathrm{L}$, respectively. Six months later, his clinical and laboratory improvements were maintained (Table 1, Fig. 3).

\section{DISCUSSION}

Neurogenic HO has been reported in $11 \%-76 \%$ of patients with traumatic brain injury, of which $10 \%-20 \%$ of cases are clinically significant [7]. To decrease further complications after $\mathrm{HO}$, early diagnosis and intervention are crucial [8].

All our three patients complained of spontaneous pain or pain aggravated by physical therapy of the involved joint. In addition, their BALP levels were elevated in all cases, reflecting the increase of bone-formation activity of HO. Pain is common with $\mathrm{HO}$ and it is associated with increased HO activity [9]. RT can effectively block the earliest steps after HO by arresting osteoblast differentiation from pluripotent mesenchymal cells [10]. In addition, RT may decrease the pain associated with inflammatory reaction around the site of HO. It might be involved in the ablation of pain receptors [5]. In this case series, RT was conducted at the course of their clinical and laboratory deterioration (ALP, BALP). All three patients showed clinical and laboratory improvement immediately after RT (Table 1, Fig. 3). Therefore, the improvement can be interpreted as the effect of RT rather than natural progress or other influences.

Few reports are available on the effects of RT in established neurogenic HO. We used the same RT protocol 
that Schaeffer and Sosner [4] used to treat two cases of established HO with pain and limited ROM of the involved hips and right elbow. In those cases, immediately after the RT, the pain was decreased, the ROM was increased, and the serum ALP level was decreased. Jang et al. [6] have also reported that RT can decrease pain and serum ALP level while increasing the ROM of treated joints in a case of established HO. Their patient showed pancytopenia after the RT but recovered to within normal range at 1 month after RT [6]. Similarly, in one of our cases (case 2), his serum white blood cell count was decreased after RT but had normalized at 1 month after RT.

In our case series, the ROM of the involved joints was improved somewhat and the pain severity was decreased after RT. Such improvements were maintained during the 6-month follow-up period. Their serum ALP and BALP levels were also decreased dramatically immediately after RT in all three cases. Such decrease was maintained throughout the follow-up period. Post-treatment X-rays revealed no further growth of the HO. Our results suggest that RT can be used as safe and effective method to immediately decrease pain and activity of neurogenic established HO. Such effect can maintain for 4-6 months after RT.

\section{CONFLICT OF INTEREST}

No potential conflict of interest relevant to this article was reported.

\section{REFERENCES}

1. Kaplan FS, Glaser DL, Hebela N, Shore EM. Hetero- topic ossification. J Am Acad Orthop Surg 2004;12:11625.

2. Orzel JA, Rudd TG. Heterotopic bone formation: clinical, laboratory, and imaging correlation. J Nucl Med 1985;26:125-32.

3. Broyles DL, Nielsen RG, Bussett EM, Lu WD, Mizrahi IA, Nunnelly PA, et al. Analytical and clinical performance characteristics of Tandem-MP Ostase, a new immunoassay for serum bone alkaline phosphatase. Clin Chem 1998;44:2139-47.

4. Cipriano C, Pill SG, Rosenstock J, Keenan MA. Radiation therapy for preventing recurrence of neurogenic heterotopic ossification. Orthopedics 2009;32:687-9.

5. Schaeffer MA, Sosner J. Heterotopic ossification: treatment of established bone with radiation therapy. Arch Phys Med Rehabil 1995;76:284-6.

6. Jang SH, Shin SW, Ahn SH, Cho IH, Kim SH. Radiation therapy for heterotopic ossification in a patient with traumatic brain injury. Yonsei Med J 2000;41:536-9.

7. Garland DE. Clinical observations on fractures and heterotopic ossification in the spinal cord and traumatic brain injured populations. Clin Orthop Relat Res 1988;233:86-101.

8. Sullivan MP, Torres SJ, Mehta S, Ahn J. Heterotopic ossification after central nervous system trauma: a current review. Bone Joint Res 2013;2:51-7.

9. Mavrogenis AF, Soucacos PN, Papagelopoulos PJ. Heterotopic ossification revisited. Orthopedics 2011; 34:177.

10. Balboni TA, Gobezie R, Mamon HJ. Heterotopic ossification: pathophysiology, clinical features, and the role of radiotherapy for prophylaxis. Int J Radiat Oncol Biol Phys 2006;65:1289-99. 